\title{
A Slowly Developed Severe Cutaneous Adverse Reaction to Idelalisib
}

L Huilaja $^{1}$, O Lindgren ${ }^{2}$, M Soronen ${ }^{1}$, T Siitonen $^{3}$, K Tasanen $^{1}$

${ }^{1}$ PEDEGO Research Unit, University of Oulu; Department of Dermatology and Medical Research Center Oulu, Oulu University Hospital, Oulu, Finland

${ }^{2}$ Cancer Research and Translational Medicine Research Unit, University of Oulu; Department of Pathology, Oulu University Hospital, Oulu, Finland

${ }^{3}$ Department of Medicine, Oulu University Hospital, Oulu, Finland

Running title: Severe Cutaneous Adverse Reaction to Idelalisib

Word count: $598 / 600$

Figures: 2

Tables: 0

Funding: None

Conflict of interest: Authors declare no conflict of interest

Corresponding author:

Laura Huilaja

Aapistie 5A, 90020 Oulu, Oulu, Finland

laura.huilaja@oulu.fi

tel. +35883760, fax. +35883153801 
Intracellular signal mediator phosphatidylinositol-3-kinase (PIK3K) $-\delta$, an isoform of PIK3K, is expressed in hematopoietic cells especially in lymphoid lineage 1 . Idelalisib is a novel PIK3K- $\delta$ targeted kinase inhibitor which is approved for relapsed follicular B-cell non-Hodgkin lymphoma as a monotherapy and in combination with rituximab, an anti-CD20 antibody, for relapsed chronic lymphocytic leukemia (CLL) 2 . Only one case describing the clinical features of severe cutaneous adverse reaction (SCAR) of idelalisib in detail has been previously published 3. Here we expand the clinical picture of SCAR caused by idelalisib with its histological profile.

After informed consent, we report a 61-year-old male with hypertension and hypercholesterolemia. At the age of 59 the diagnosis of asymptomatic CLL was made. Later he developed lymphadenopathy, leukocytosis and anemia. TP53-mutation was found and idelalisib (Zydelig ${ }^{\circledR}$; 150mg twice a day) - rituximab (Mabthera ${ }^{\circledR} ; 375-500 \mathrm{mg} / \mathrm{m}^{2}$, 2-4 weeks interval) -combination was started as his first treatment. In addition, oral sulfa-trimethoprim was dosed for pneumocystis pneumonia prevention. Three months later he reported having a fluctuating, non-pruritic rash on his upper arms and trunk that had lasted already for two months. Sulfa-trimethoprim was withdrawn and replaced by an inhalable pentamidine. Within a month, despite of drug replacement, his skin symptoms got worse and alanine aminotransferase (ALT) increased up to $291 \mathrm{U} / \mathrm{I}$ (normal $<50 \mathrm{U} / \mathrm{I})$. Idelalisib was discontinued and he was sent for a dermatological consultation. He had some macular erythematous lesions with partial desquamation over his sides and back (Fig. 1a) and superficial peeling in palms (Fig. 1b). Skin biopsy showed histological features suggestive for exanthematous drug reaction (Fig. 2). As a treatment a potent topical steroid was administered. After a week, his liver enzymes were improved (ALT $145 \mathrm{U} / \mathrm{I}$ ), but skin symptoms proceeded. His skin was erythrodermic and desquamating (Fig. 1C-e), but Nikolsky sign was negative. No mucosal lesions 
were found. He was admitted to the hospital and oral prednisolone $(0.33 \mathrm{mg} / \mathrm{kg})$ and potent topical steroids were administered with supportive skin care. Skin symptoms rapidly improved and oral prednisolone was tapered within two weeks. Because of the grade 3 adverse reaction, idelalisib has not been restarted hereafter. The treatment of choice in his next CLL relapse will be venetoclax.

In idelalisib efficacy studies on CLL, $10-22 \%$ of patients were reported to develop rash in monotherapy 4,5 and $58 \%$ in combination with rituximab, respectively 6 . Most skin reactions (97\%) were classified mild, but a few grade $\geq 3$ reactions with maculopapular eruption and generalized exfoliative dermatitis were reported 7. A recent case report described a man with grade 3 exfoliative and erythematous rash on wide areas of the body and mucosal membranes appearing three months after the start of idelalisib 3. In our case the symptoms progressed first during the first couple months quite slowly, until SCAR finally manifested in one week. Our case did neither show any mucosal symptoms.

By August 2017, 222 idelalisib-associated suspected skin reactions have been reported in European database of suspected adverse drug reactions ( $\underline{w w w . a d r r e p o r t s . e u})$. Most cutaneous adverse reactions were reported as rash $(n=32)$ or exfoliative dermatitis $(n=12)$. However, descriptions have varied from psoriasis to Stevens-Johnson syndrome $(n=5)$. In any of the previous skin toxicity reports, no histological analysis has been performed. In our case, skin biopsy showed an exanthematous drug reaction but specific histological findings could not be found. 
Our case demonstrates that slowly progressing cutaneous toxicity of idelalisib may worsen in few days up to SCAR even after withdrawal of the culprit treatment. At the moment, the mechanism of this type of idelalisib-induced SCAR is not understood, and the treatment of these reactions is mainly supportive with the discontinuation of the drug 8.

\section{References}

1. Wiestner A. BCR pathway inhibition as therapy for chronic lymphocytic leukemia and lymphoplasmacytic lymphoma. Hematology Am Soc Hematol Educ Program 2014;2014:125-34.

2. Shah A, Mangaonkar A. Idelalisib: A Novel PI3Kdelta Inhibitor for Chronic Lymphocytic Leukemia. Ann Pharmacother 2015;49:1162-70.

3. Gabriel JG, Kapila A, Gonzalez-Estrada A. A Severe Case of Cutaneous Adverse Drug Reaction Secondary to a Novice Drug: Idelalisib. J Investig Med High Impact Case Rep 2017;5:2324709617711463.

4. Furman RR, Sharman JP, Coutre SE, Cheson BD, Pagel JM, Hillmen P, et al. Idelalisib and rituximab in relapsed chronic lymphocytic leukemia. N Engl J Med 2014;370:997-1007.

5. Brown JR, Byrd JC, Coutre SE, Benson DM, Flinn IW, Wagner-Johnston ND, et al. Idelalisib, an inhibitor of phosphatidylinositol 3-kinase p110delta, for relapsed/refractory chronic lymphocytic leukemia. Blood 2014;123:3390-7.

6. O'Brien SM, Lamanna N, Kipps TJ, Flinn I, Zelenetz AD, Burger JA, et al. A phase 2 study of idelalisib plus rituximab in treatment-naive older patients with chronic lymphocytic leukemia. Blood 2015;126:2686-94.

7. Miller BW, Przepiorka D, de Claro RA, Lee K, Nie L, Simpson N, et al. FDA approval: idelalisib monotherapy for the treatment of patients with follicular lymphoma and small lymphocytic lymphoma. Clin Cancer Res 2015;21:1525-9.

8. de Weerdt I, Koopmans SM, Kater AP, van Gelder M. Incidence and management of toxicity associated with ibrutinib and idelalisib: a practical approach. Haematologica 2017;102:1629-39. 


\section{Figure 1}

Clinical presentation of the severe cutaneous adverse reaction to idelalisib. Macular lesions in sides (a) and superficial peeling in palms (b) at the first visit to the dermatologist after idelalisib was discontinued. A week later erythrodermic and widely desquamating skin eruption was seen in the trunk (c-d) and skin peeling in palms (e).

\section{Figure 2}

In the histological analysis (Haematoxylin-eosin staining, 20x magnification) the keratotic layer varied from thin normal basket weave-keratosis to mild hyperkeratosis and focal parakeratosis. Mild acanthotic thickening, focal spongiosis and some spongiotic lymphocyte-neutrophil containing pustules as well as inflammatory cell exocytosis in the epidermis were seen. In the dermis, the mostly perivascular inflammatory infiltrate was composed mainly of lymphocytes, and histiocytes but also some eosinophils were seen. 